\title{
Investigation of the influence of ambient temperature, atmospheric pressure and water vapour pressure on epistaxis admission rate*
}

\author{
V.M. Reddy ${ }^{1}$, O. Judd ${ }^{2}$, H. Khalil ${ }^{3}$ \\ 1 ENT Department, Royal Cornwall Hospitals NHS Trust, Truro, Cornwall, United Kingdom \\ 2 ENT Department, University Hospitals Leicester NHS Trust, Leicester, United Kingdom \\ 3 ENT Department, Plymouth Hospitals NHS Trust, Plymouth, Devon, United Kingdom
}

\begin{abstract}
SUMMARY Background: To investigate the relationship between weather variables (atmospheric pressure, temperature, water vapour pressure) and epistaxis admission rates at Derriford Hospital, Plymouth, United Kingdom.

Methodology: Retrospective observational study using hospital inpatient information databases to identify all patients admitted with epistaxis from April 1999 to March 2009 inclusive. Meteorological data for the same period was retrieved from the University of Plymouth Meteorological Archive. Epistaxis admissions were investigated for correlation with weather variables using Pearson correlation, and stepwise multiple regression analysis was performed. Results: During the study period there were 1071 admissions (978 patients) (501 males (mean age 64 years) and 477 females (mean age 72 years)). Less than $1 \%$ of the variance in epistaxis admissions is explained by the maximum temperature only. Temperature and water vapour pressure demonstrated statistically significant association with epistaxis admission rates.

Conclusions: Though there is statistical significance in the association of some weather variables and epistaxis admission rates, the findings are not practically relevant $(<1 \%$ variance). The results of this study do not indicate a need for revision of current healthcare resource allocation.
\end{abstract}

Key words: epistaxis, temperature, climate, meteorological factors, hospital admission

\section{INTRODUCTION}

Epistaxis affects $50-60 \%$ of the population at some point in their lives. It affects all ages to varying degrees, with a bimodal peak of incidence at 15-25 and 45-65 years of age ${ }^{(1)}$. Hospital admission is necessary for $6 \%$ of these patients ${ }^{(1)}$. The daily financial implications of an inpatient stay for epistaxis has been estimated at $£ 260$ per day ${ }^{(1)}$. Several studies in the past have aimed to establish whether there is a seasonal variation in the presentation rates of epistaxis, but the results have not been conclusive $^{(2-5)}$.

Danielides investigated the association of epistaxis admission rates with atmospheric pressure, humidity and temperature in Greece, which the authors suggest may not be reflective of other regions with different climates ${ }^{(3)}$. We aim to investigate whether there is any relationship between weather variables and epistaxis admission rates in our population in the South West of England.

\section{MATERIALS AND METHODS \\ Patients}

We conducted a retrospective observational study using information retrieved from hospital and meteorological databases. Details regarding inpatient episodes were retrieved from hospital information systems for all epistaxis admissions to Derriford Hospital, Plymouth from the $1^{\text {st }}$ of April 1999 to the $31^{\text {st }}$ of March 2009 inclusive. Patients were excluded if taking anticoagulant medication at the time of admission, or if they had a history of haematological disorders or hereditary haemorrhagic telangiectasia. We also excluded readmissions within 28 days (we considered these to represent incomplete treatment as opposed to recurrence of epistaxis).

\section{Weather data}

Daily readings of ambient temperature, atmospheric pressure and humidity were obtained from the Plymouth University Meteorological Archive. The humidity recordings were used to calculate water vapour pressure using the Clausius-Clapeyron equation, as this is believed to be more clinically relevant than 
relative humidity ${ }^{(3)}$. Previous studies have also relied on meteorology data from local universities ${ }^{(3)}$, or from local airport weather records ${ }^{(2,4,5)}$. We considered the minimum, maximum, range (maximum minus minimum), mean and standard deviation (as a measure of variance) of ambient temperature, atmospheric pressure and water vapour pressure.

\section{Statistical analysis}

Statistical analysis was performed using SPSS 13.0 for Windows (SPSS Inc., Chicago, IL, USA). Independent samples T-test was used to compare ambient temperature between days on which epistaxis acute admissions were and were not encountered. Chi-square test was used to compare atmospheric pressure and water vapour pressure as these variables have a non-parametric distribution. There was no linear association between any of the variables. It was therefore appropriate to perform stepwise multiple regression analysis to give an indication of the percentage of variance of epistaxis admission rates due to the aforementioned weather variables.

\section{Ethical issues}

The study protocol was submitted to the institutional research review body and the regional ethics committee. Following review by the National Research Ethics Service, it was deemed that this study did not require ethics committee review.

\section{RESULTS}

During the study period, we identified 1071 admissions (978 patients) for epistaxis which fulfilled the inclusion criteria for the study. The study group consisted of 501 males (mean age 64 years, range 18-101 years, SD 17.5) and 477 females (mean age 72 years, range 18-101 years, SD 15.8). The monthly epistaxis admission totals and average temperature are shown in Figure 1.

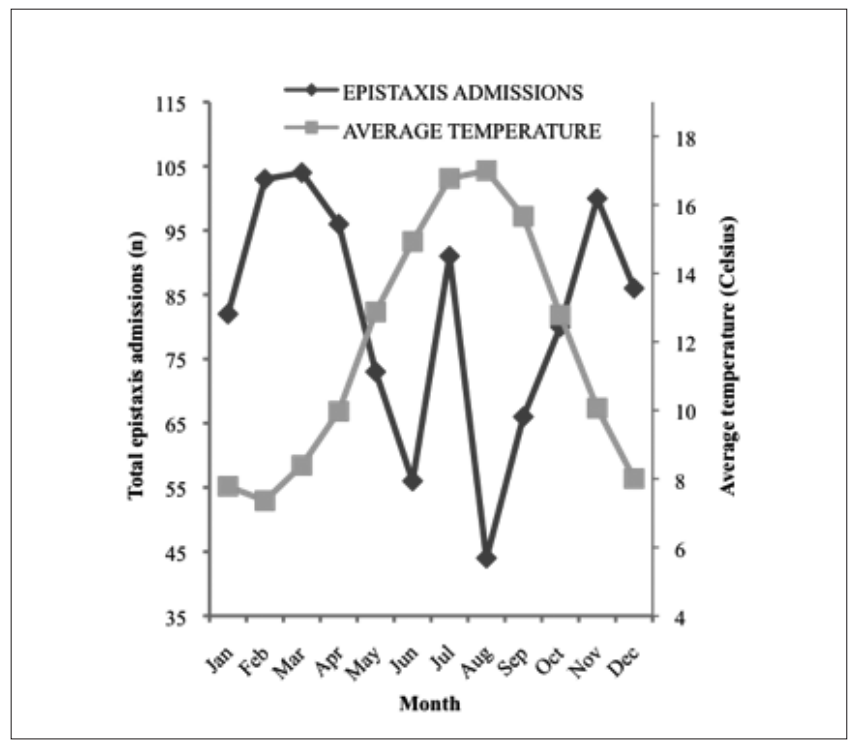

Figure 1. Monthly epistaxis admission totals and average temperature (April 1999 to March 2009). Line diagram demonstrating monthly epistaxis admissions with average temperature.
There is a sharp rise in emergency admissions in July. This is likely explained by the increased population due to tourism in the South West of England during that period. This trend is also reflected in non-epistaxis emergency admissions encountered by the ENT department (Figure 2). We therefore analysed the data in entirety, and also by excluding data for July to correct for the transient increase in population. Pearson correlation coefficients of total number of epistaxis admissions per day versus weather variables are shown in Table 1.

Multivariate regression analysis revealed that less than $1 \%$ of the variance in epistaxis admissions is explained by the maximum temperature only (including July data $\mathrm{R}=0.06$, excluding July data $\mathrm{R}=0.08$ ). There was no statistically significant improvement from incorporating any other variables which were therefore excluded from the model.

\section{DISCUSSION}

In this study, maximum temperature had a negative linear correlation with epistaxis admission rates accounting for $0,6 \%$ to $0,8 \%$ of variability. We do not believe this to be practically relevant as the values are small, thereby not requiring revision of current healthcare resource allocation.

These findings are broadly consistent with other published results that have identified a negative correlation between epistaxis admission rate and ambient temperature. Nunez et al. demonstrated an inverse linear relationship between monthly admissions for epistaxis and monthly ambient temperature (with more admissions during colder months) ${ }^{(4)}$. Stepwise multiple regression analysis revealed that $65 \%$ of the variance in monthly admissions could be explained by mean temperature ${ }^{(4)}$. To correct for the possible source of error in using

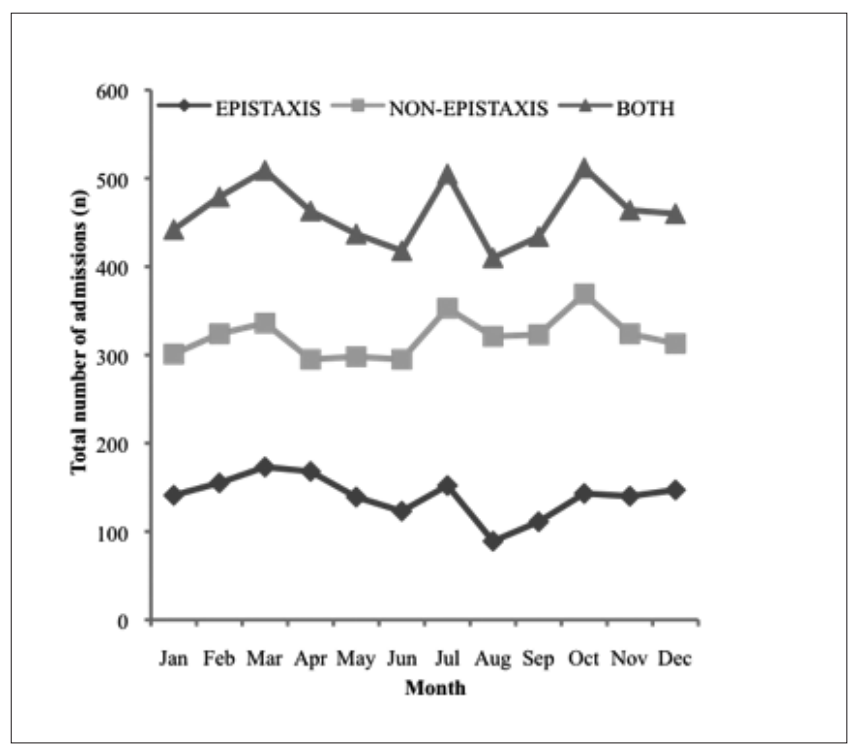

Figure 2. Monthly ENT emergency admissions (April 1999 to Mach 2009). Line diagram demonstrating monthly epistaxis admissions, nonepistaxis admissions and total ENT emergency admissions. 
Table 1. Pearson correlation coefficients of number of epistaxis admissions and weather variables.

\begin{tabular}{lcccccc}
\hline & \multicolumn{2}{c}{$\begin{array}{c}\text { Weather variables } \\
\end{array}$} & \multicolumn{2}{c}{ Atmospheric pressure } & \multicolumn{2}{c}{ Water vapour pressure } \\
\hline & \multicolumn{2}{c}{ Ambient temperature } & \multicolumn{2}{c}{ Excluding July } \\
\hline Aspect of variable & & & & & & \\
\hline Minimum & $-0.045^{*}$ & $-0.063^{*}$ & 0.020 & 0.020 & $-0.042^{\wedge}$ & $-0.060^{*}$ \\
Maximum & $-0.060^{*}$ & $-0.078^{*}$ & 0.021 & 0.025 & $-0.055^{*}$ & $-0.073^{*}$ \\
Range (max-min) & $-0.036^{\wedge}$ & $-0.035^{\wedge}$ & 0.001 & 0.009 & $-0.039^{\wedge}$ & $-0.044^{\wedge}$ \\
Average (mean) & $-0.055^{*}$ & $-0.074^{*}$ & 0.021 & 0.023 & $-0.048^{*}$ & $-0.067^{*}$ \\
Standard deviation & $-0.046^{\wedge}$ & $-0.043^{\wedge}$ & 0.002 & 0.012 & $-0.043^{\wedge}$ & $-0.041^{\wedge}$ \\
\hline
\end{tabular}

$*=$ statistically significant values at $95 \%$ confidence level, ${ }^{\wedge}=$ statistically significant values at $99 \%$ confidence level.

average monthly temperatures by Nunez et al., Tomkinson et al. investigated 1211 epistaxis admissions in Cardiff ${ }^{(5)}$. A nonlinear inverse relationship between temperature and admission rate was identified (patients were admitted on days that were $0.6^{\circ} \mathrm{C}$ colder than the average temperature for the time period, $\mathrm{p}<0.05$, students t-test). Danielides et al. investigated the influence of temperature, atmospheric pressure and humidity on the frequency of epistaxis presentations ${ }^{(3)}$. The study included 701 patients treated in the University of Ioannina hospital in Greece from 1995 to 1996, of which 170 were admitted. Multiple regression analysis revealed that during colder months, $17 \%$ of the variance was explained by these meteorological variables, compared with 9\% during warmer months. Thus weather variables were felt to significantly influence epistaxis frequency. However, Bray et al. conducted a retrospective analysis of epistaxis presentations to a London hospital from 1997 to 2002, involving 1373 patients (only 386 were admitted) (2). The months with the highest incidence of spontaneous epistaxis were November and June. The authors therefore concluded that admission or presentation of patients with epistaxis did not correlate with ambient temperature.

The influence of weather variables on epistaxis therefore is not clear, but it would appear that most of the studies have concluded that colder temperatures are associated with epistaxis. The variability in results between the studies is quite considerable however. This may be due to the differences in population characteristics and comorbidities in different regions, or it may be reflective of local climatic differences. The results therefore cannot be generalised to other people or regions.

Whilst several studies, including our own, have demonstrated an association of epistaxis admission with colder temperatures, it is unclear as to why cold temperatures should have this effect. Cold temperatures may be an indicator of other factors such as upper respiratory tract infections (URTIs) and hypothermic coagulopathy. It is commonly known that URTIs are more prevalent during the winter; Eccles suggested that colder temperatures impair nasal mucosal defences by hindering mucocilliary clearance and reducing cellular immunity ${ }^{(6)}$, thus colder temperatures predispose to the development of URTI. It is therefore plausible that the rise in epistaxis admis- sions during winter may reflect URTI rather than a direct influence of cold temperature. It has also been suggested that hypothermia affects coagulation. Rundgren and Engstrom investigated blood coagulability at varying temperatures ( 25 to $40^{\circ} \mathrm{C}$ ) using thromboelastography ${ }^{(7)}$. They demonstrated that coagulation was increasingly impaired as temperature reduced

(7). The elderly are particularly vulnerable to epistaxis for several reasons. Elderly patients' blood vessels demonstrate morphological changes including diminished elasticity and increased smooth muscle tone which increases vascular stiffness and thereby affects normal function ${ }^{(8)}$. They also have impaired thermoregulation, probably due to an inadequate autonomic response. This compounds the effect of the cold on URTI development and on coagulability.

Epistaxis is the most common emergency encountered by ENT surgeons. The influence of weather variables on admission rates is of interest as it has implications for the allocation of healthcare resources. In this study, the most statistically significant finding was a negative linear correlation of epistaxis admission with ambient temperature, which accounted for $0,6-0,8 \%$ of variability in epistaxis admission rates. Epistaxis is a multifactorial disease and the association with cold temperature may actually reflect other associated states including increased prevalence of URTIs and hypothermic coagulopathy.

\section{AUTHORSHIP CONTRIBUTION}

$\mathrm{Mr}$ Reddy and Mr Judd were involved in gathering the data and statistical analysis. Mr Reddy produced the first draft of the paper, which was rewritten by Mr Judd and Mr Khalil. Figures and tables were produced by Mr Reddy.

\section{CONFLICT OF INTEREST}

No conflicts of interest for any of the authors.

\section{REFERENCES}

1. Ahmed A, Woolford TJ. Endoscopic bipolar diathermy in the management of epistaxis: an effective and cost-efficient treatment. Clin Otolaryngol Allied Sci. 2003; 28: 273-275.

2. Bray D, Giddings CE, Monnery P, et al. Epistaxis: are temperature and seasonal variations true factors in incidence? J Laryngol Otol. 2005; 119: 724-726. 
3. Danielides V, Kontogiannis N, Bartzokas A, et al. The influence of meteorological factors on the frequency of epistaxis. Clin Otolaryngol Allied Sci. 2002; 27: 84-88.

4. Nunez DA, McClymont LG, Evans RA. Epistaxis: a study of the relationship with weather. Clin Otolaryngol Allied Sci. 1990; 15: 49-51.

5. Tomkinson A, Roblin DG, Flanagan P, Quine SM, Backhouse S. Patterns of hospital attendance with epistaxis. Rhinology. 1997; 35: 129-131.

6. Eccles R. An explanation for the seasonality of acute upper respiratory tract viral infections. Acta Otolaryngol. 2002; 122: 183-191.

7. Rundgren M, Engstrom M. A thromboelastometric evaluation of the effects of hypothermia on the coagulation system. Anesth Analg. 2008; 107: 1465-1468.

8. Priebe HJ. The aged cardiovascular risk patient. Br J Anaesth. 2000; 85: 763-778.
Mr. V.M. Reddy

ENT Department

Royal Cornwall Hospitals NHS Trust

Truro

Cornwall

TR1 3LJ, United Kingdom

Tel: +44-187-2250000

E-mail: vmreddy@doctors.net.uk 